\title{
BMJ Open Effects of whole-body MRI on outpatient health service costs: a general-population prospective cohort study in Mecklenburg-Vorpommern, Germany
}

Carsten Oliver Schmidt (D) , ${ }^{1}$ Elizabeth Sierocinski, ${ }^{2}$ Sebastian-Edgar Baumeister, ${ }^{3}$ Katrin Hegenscheid, ${ }^{4,5}$ Henry Völzke, ${ }^{1,6,7}$ Jean-François Chenot (i) ${ }^{2}$

To cite: Schmidt CO, Sierocinski E, Baumeister S-E, et al. Effects of whole-body MRI on outpatient health service costs: a general-population prospective cohort study in Mecklenburg-Vorpommern, Germany. BMJ Open 2022;12:e056572. doi:10.1136/ bmjopen-2021-056572

- Prepublication history for this paper is available online. To view these files, please visit the journal online (http://dx.doi. org/10.1136/bmjopen-2021056572).

Received 23 August 2021 Accepted 15 December 2021

D) Check for updates

(C) Author(s) (or their employer(s)) 2022. Re-use permitted under CC BY-NC. No commercial re-use. See rights and permissions. Published by BMJ.

For numbered affiliations see end of article.

Correspondence to Dr Carsten Oliver Schmidt; carsten.schmidt@unigreifswald.de

\section{ABSTRACT}

Objective Whole-body MRI (wb-MRI) is increasingly used in research and screening but little is known about the effects of incidental findings (IFs) on health service utilisation and costs. Such effects are particularly critical in an observational study. Our principal research question was therefore how participation in a wb-MRI examination with its resemblance to a population-based health screening is associated with outpatient service costs. Design Prospective cohort study.

Setting General population Mecklenburg-Vorpommern, Germany.

Participants Analyses included 5019 participants of the Study of Health in Pomerania with statutory health insurance data. 2969 took part in a wb-MRI examination in addition to a clinical examination programme that was administered to all participants. MRI non-participants served as a quasi-experimental control group with propensity score weighting to account for baseline differences.

Primary and secondary outcome measures Outpatient costs (total healthcare usage, primary care, specialist care, laboratory tests, imaging) during 24 months after the examination were retrieved from claims data. Two-part models were used to compute treatment effects.

Results In total, 1366 potentially relevant IFs were disclosed to $948 \mathrm{MRI}$ participants $(32 \%$ of all participants); most concerned masses and lesions (769 participants, $81 \%$ ). Costs for outpatient care during the 2-year observation period amounted to an average of $€ 2547$ (95\% Cl 2424 to 2671) for MRI non-participants and to $€ 2839$ (95\% Cl 2741 to 2936) for MRI participants, indicating an increase of $€ 295$ (95\% Cl 134 to 456) per participant which corresponds to $11.6 \%$ (95\% Cl 5.2\% to $17.9 \%)$. The cost increase was sustained rather than being a short-term spike. Imaging and specialist care related costs were the main contributors to the increase in costs.

Conclusions Communicated findings from populationbased wb-MRI substantially impacted health service utilisation and costs. This introduced bias into the natural course of healthcare utilisation and should be taken care for in any longitudinal analyses.

\section{Strengths and limitations of this study}

$\Rightarrow$ This is the first study to report disclosed longitudinal effects of disclosed incidental whole body MRI findings on costs for outpatient services in a general population setting; the longitudinal cohort design, large sample size and the availability of groups with and without MRI participation are considerable strengths.

$\Rightarrow$ Claims data provide an appropriate approximation of outpatient costs as they are collected for reimbursement purposes.

$\Rightarrow$ Limitations concern the scope of claims data as they do not cover medication or inpatient care, and we are likely to have underestimated the total healthcare-related expenditures compared with a clinical scenario because only selected findings were disclosed to participants.

$\Rightarrow$ The small subgroup of privately insured participants is not represented.

$\Rightarrow$ Non-randomised allocation to MRI participation reduces the comparability of participants and non-participants.

\section{INTRODUCTION}

Screening with whole-body MRI (wb-MRI) may detect asymptomatic disease at an early stage thus improve treatment outcome, ${ }^{1}$ but might also cause unnecessary psychosocial distress, medical interventions and costs due to irrelevant findings. ${ }^{23} \mathrm{~Wb}$-MRI has evolved into a key examination tool in state-of-the-art population research. ${ }^{4-7}$ It produces a large number of incidental findings (IFs), a large proportion of which represent masses and lesions. ${ }^{2-11}$ Although a minority may benefit from IFs in a general population approach, ${ }^{1}$ there is uncertainty around the clinical significance of a large proportion of these IFs. There is a high risk of false-positives, overtesting and overdiagnosis. ${ }^{12} 13$ Only few 
malignancies were newly detected despite a $42 \%$ increase in biopsies after participation in Study of Health in Pomerania (SHIP) ${ }^{14}$ and no positive effects on quality of life resulted.$^{15}$ In contrast, there is clear evidence of shortterm adverse consequences such as psychological distress due to disclosed findings. ${ }^{16}$

To the best of our knowledge, there is no prospective evidence on the effects of wb-MRI IFs in a general population setting on ambulatory health service utilisation and associated costs. Such data are needed to guide the appropriate handling of wb-MRI screening results in research and clinical practice.

Our principal research question was therefore how participation in a wb-MRI with resemblance to a population-based health screening is associated with outpatient service utilisation and costs. MRI nonparticipants served as a quasi-experimental control group with propensity score weighting to account for baseline differences. We hypothesised that participation in the MRI examination would lead to increased costs in ambulatory healthcare. Furthermore, we assume that there are differential effects on different types of ambulatory services.

\section{METHODS}

\section{Study design and sample}

SHIP is a population-based project consisting of two independent prospective cohort studies, SHIP and SHIPTrend. Participants were sampled from the counties of North and East Western Pomerania and the cities of Greifswald and Stralsund in Germany. ${ }^{7}$ Participants were between 20 and 79 years of age at the date of sampling and had the target region listed as their primary place of residence. The aim in both cohorts was to recruit a representative general population sample. Clinical status was of no relevance for inclusion. Participants received no payment beyond reimbursement for travel costs.

A two-stage sampling scheme was adopted from the German WHO's MONICA (monitoring trends and determinants in cardiovascular disease) Project for the first cohort. ${ }^{17}$ Out of 6265 eligible individuals of the first cohort, 4308 (2192 women) participated at baseline (response $68.8 \%){ }^{18}$ Baseline examinations were performed from 1997 to 2001 (SHIP0 ). Follow-up examinations took place between 2002 and 2006 (SHIP-1, N=3300) and between 2008 and 2012 (SHIP-2, $\mathrm{N}=2333$ ).

A second cohort (SHIP-Trend) was established in 2008 from a stratified sample of 10000 adults, drawn from the central population registry. Examinations took place until 2012. Of the net sample of 8826, after exclusion of deceased and relocated participants, 4420 (2275 women) participated (response $50.1 \%$ ).

Of the 6753 participants in SHIP-2 and SHIP-Trend, 6312 had statutory health insurance $(93 \%)$. Subjects ineligible for MRI participation for reasons such as claustrophobia, metal implants or pregnancy were excluded from analyses ${ }^{15}$ because treatment effects could not be

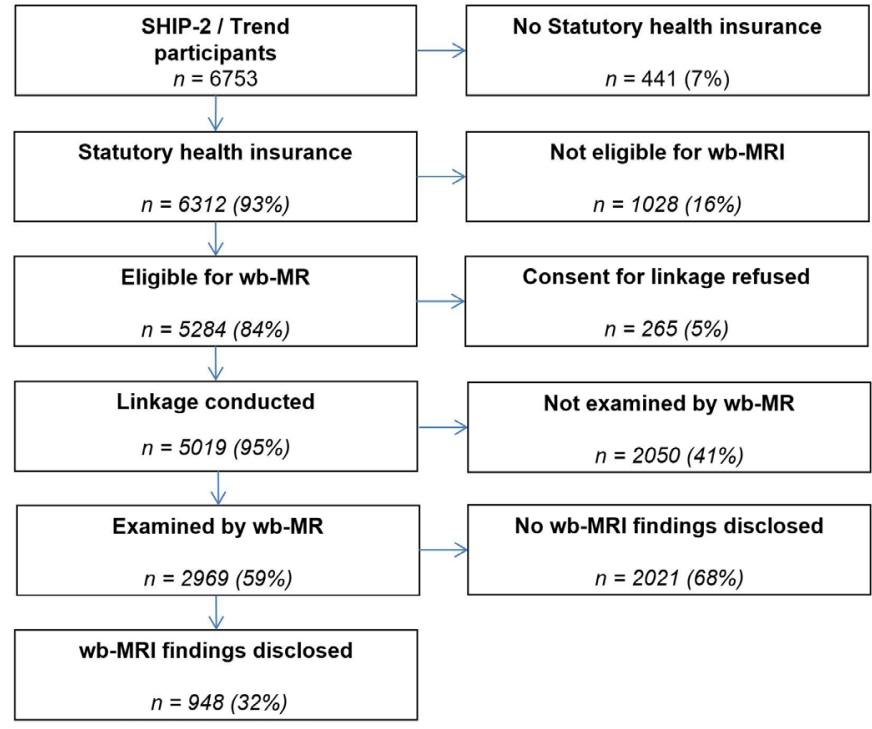

Figure 1 Study flow chart. SHIP, Study of Health in Pomerania; wb-MRI, whole-body MRI.

meaningfully computed for this group $(n=1028)$. Of the resulting 5284 participants, $265(5 \%)$ refused linkage with statutory health insurance. Our analyses included the remaining 5019 participants (figure 1). The follow-up period for claims data was 2 years after SHIP participation.

\section{MRI examination}

All SHIP participants were invited to take part in the MRI examination and received relevant written educational material. During a medical interview before the examination, a radiologist described the handling of IFs and conditions for disclosure ${ }^{8}$. The methods descriptions in MRI examination and Disclosure of IFs have been taken from previous SHIP publications. ${ }^{1415}$

All wb-MRI were acquired on a 1.5-Tesla system (Magnetom Avanto; Siemens Medical Solutions, Erlangen, Germany). The wb-MRI protocol was identical for all participants and included a plain wb-MRI and detailed imaging of the head, neck, chest, abdomen, pelvis and spine. Men had the option of contrast-enhanced cardiac MRI and MR angiography, and women had the option of cardiac MRI and contrast-enhanced MR mammography. The complete imaging protocols have been described previously. ${ }^{819}$

Findings and anatomical variants were documented in a standardised reading protocol. The radiologists reading the scans had no access to the participants' clinical information. Scan reading was performed using a digital picture archiving and communication system (IMPACS ES V.5.2, AGFA Healthcare, Mortsel, Belgium). First-line reading was performed by two independent radiology residents. A third reader, a senior radiologist, resolved disagreements.

The MRI examination was entirely financed by SHIP study funding and did not contribute to billing costs represented in the claims data.

\section{Disclosure of IFs}

A standardised protocol regulated the handling of wb-MRI IFs. Findings were classified into three categories: 
category I comprised normal or common findings in asymptomatic individuals (eg, anatomical variants, old brain infarcts, disc herniation, sinusitits). Category II findings were abnormalities of potential clinical relevance. Category III findings required immediate referral. Category II findings were disclosed in writing via post after approval by an interdisciplinary advisory board (eg, breast lesion $\geq$ BIRADS 3 , adrenal tumour $>10 \mathrm{~mm}$, lung nodule $>4 \mathrm{~mm}$, chronic pancreatitis, internal carotid artery stenosis). Category III findings were disclosed immediately to the participant (eg, acute brain infarction, intracranial haemorrhage, lobar pneumonia, bone fracture). A detailed description of this protocol has been provided elsewhere. ${ }^{8}$ Our analyses are based on category II and III findings.

Health-related findings from other examinations such as blood testing, blood pressure measurements, somatometry, ultrasound and cardiovascular examinations were also disclosed to SHIP participants. ${ }^{7}$

\section{Claims data}

Claims data from the regional Association of Statutory Health Insurance Physicians included billing codes for medical and technical services (eg, imaging) and costs for outpatient care. Claims data do not include medication costs or services like emergency department attendances. Germany has a mixed billing system with capitation and fee-for-service models for specific services. We computed costs per quarter (billing period) for the two quarters prior to the examination, the quarter during which the examination took place and the eight quarters following the examination. We distinguished between (1) total outpatient costs, (2) primary care and prevention, (3) specialist care, (4) laboratory work and (5) imaging. All costs related to pregnancy and births were excluded, as pregnancy was an exclusion criterion for MRI participation.

\section{Statistical analyses}

We used two-part models to analyse cost variables with their zero inflated distribution. ${ }^{20}$ Each two-part model comprised a multivariable logistic regression for any healthcare service provision and a generalised linear model with a log-link and a gamma distribution for the height of costs. Models used the predictors time, MRI participation (yes vs no), and the interaction term to model average treatment effects. Propensity score reweighting was applied to balance the distribution of relevant covariates between MRI participants and nonparticipants. ${ }^{2122}$

Logistic regression models were used to estimate propensity scores with the following predictors: costs for total healthcare, primary care and prevention, specialist care, laboratory work and imaging in the two quarters prior to the SHIP-2/SHIP-Trend examination, as well as age, sex, level of education ( $<10$ years vs $\geq 10$ years), marital status, current employment, smoking status and quality of life using the short form 12-item mental health component summary score and physical health component summary score. ${ }^{23}$ Resulting weights ranged from 1.0 to 13.5 (mean: 2.5; SD 0.8). Standardised mean differences (SMD) of all baseline variables after weighting were close to zero ( $\mathrm{min}:-0.007,10 \%$ percentile: -0.004 , median: $0.90 \%$ percentile: 0.003, max: 0.005). Balance was also checked based on distributional properties of all baseline variables and all statistical interaction effects (SMD distribution: min: $-0.045,10 \%$ percentile: -0.019 , median: $-0.002,90 \%$ percentile: 0.009 , max: 0.045$)$.

Item missingness is reported in the legend of table 1. Cost data were unavailable for $7.4 \%$ of all quarters. For $4.7 \%(\mathrm{~N}=238)$ no doctoral visit was coded at all during the observation period. Given the plausibility of a subgroup not presenting to ambulatory care regularly, we set all types of costs in these quarters to $€ 0$ as a coded visit is a prerequisite for costs. Nevertheless, we cannot be certain that data for some participants who did present to the doctor were missing. We therefore used multiple imputation by chained equations as a sensitivity analysis to impute missing information on costs and coded neoplasms. These results did not lead to different conclusions and are thus not presented.

Two-sided tests were applied throughout. Analyses were conducted in Stata V.14 (Stata Corp.). Figures were generated using Microsoft PowerPoint.

\section{Patient and public involvement}

Patients, study participants and the public were not directly involved in the design, conduct and reporting of this study.

\section{RESULTS}

\section{Sample characteristics and IFs}

MRI non-participants were on average 3 years older, had a lower level of education, were more often unemployed, smokers and less often married compared with MRI participants (table 1). Total ambulatory costs were similar in both groups and quality of life scores were slightly lower among non-MRI participants.

In total, 1366 IFs of potential clinical relevance were disclosed to 948 participants (32\%). Of these, 769 participants $(81 \%)$ received a finding related to masses and lesions, corresponding to $26 \%$ of all MRI participants. A more detailed overview of the structure and type of wb-MRI findings and the affected organs has been provided previously. ${ }^{8}$

\section{Descriptive course of outpatient costs}

The course of outpatient costs is displayed stratified for MRI non-participants, MRI participants with disclosed findings and MRI-participants who did not receive findings (figure 2). The latter two groups were distinguished because a marked increase in costs after the SHIP examination was only expected among those with a disclosed finding. Increases occurred in all studied groups but peaks were highest in the quarter after the SHIP examination 
Table 1 Sample characteristics of MRI participants and MRI non-participants

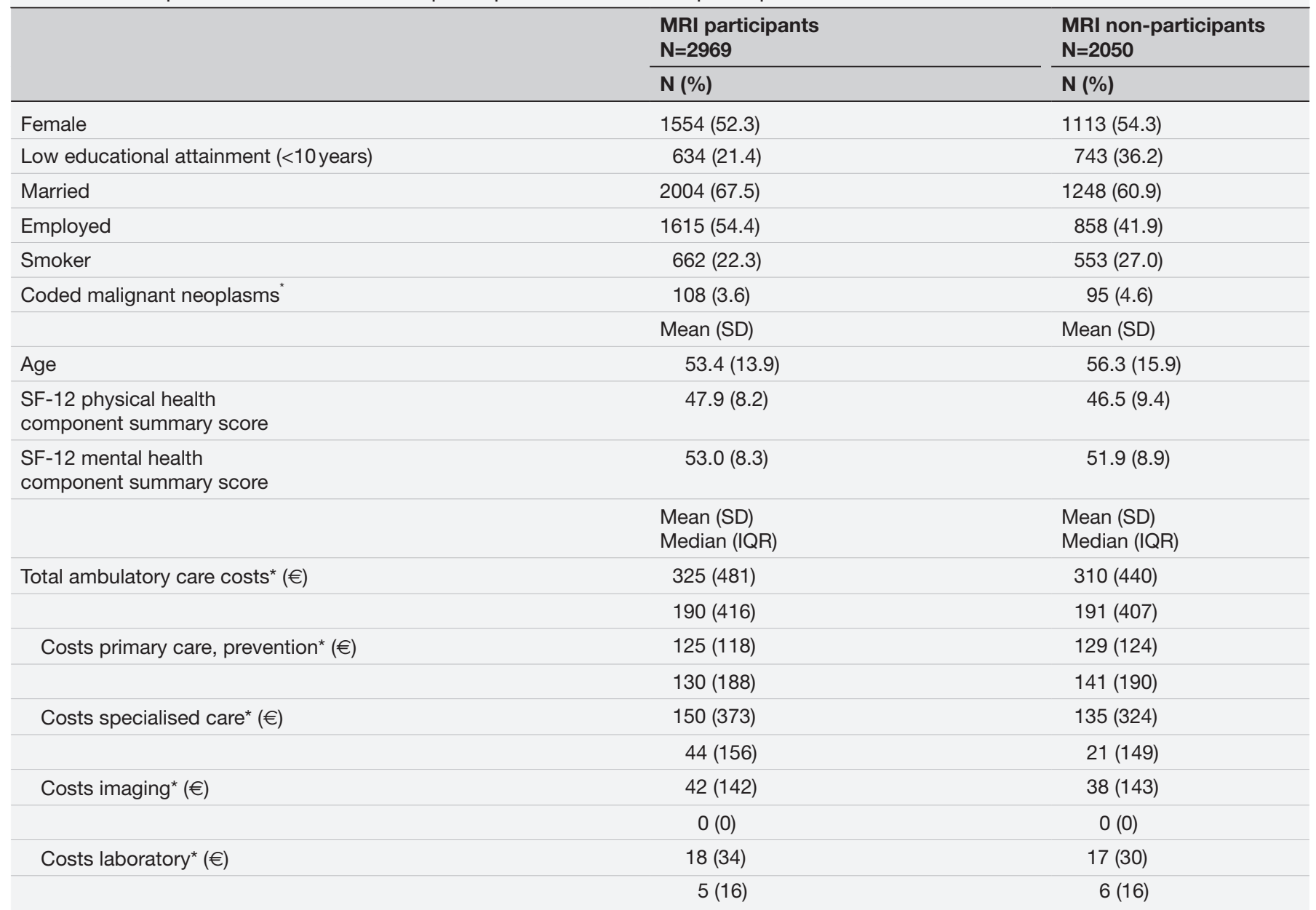

Item missingness: age, sex: 0\%; educational status: 0.2\%; marital status: 0.2\%; employment status: 0.3\%; smoking: 0.4\%; short form 12-item (SF12): $0.3 \%$; cost data: $7 \cdot 4 \%$. Presented are unimputed variables with the exception of cost data with a 0 imputation, as described in methods. ${ }^{*}$ Costs refer to the quarter prior to the whole-body examination.

among MRI participants with disclosed findings (figures 2 and 3). The relative increase was largest for imagingrelated costs, which is more than doubled (figure 3). While decreasing after an initial peak, total costs among
MRI participants with disclosed findings did not return to the initial level but remained higher (figures 2 and 3 ).
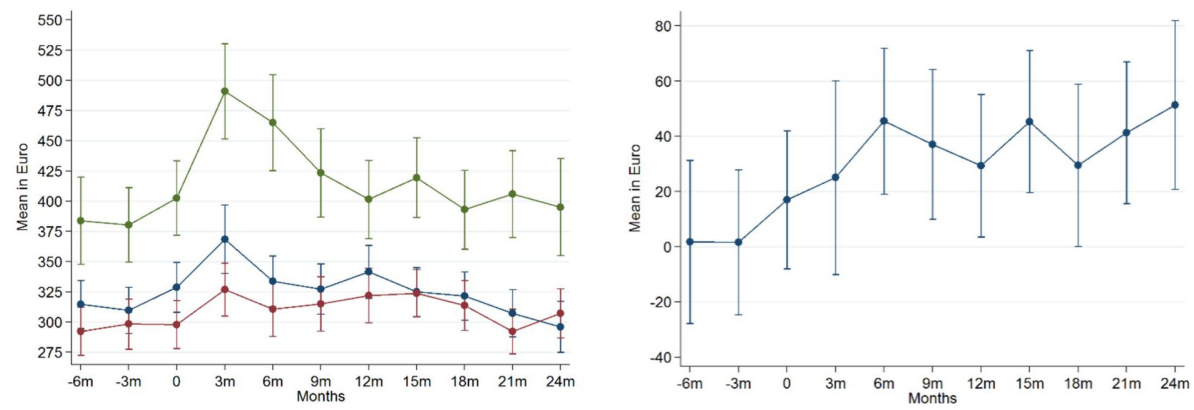

Figure 2 Course of total costs. "0" is the reference quarter in which the MRI examination took place. The left column displays the descriptive course for the outcomes comparing MRI participants with disclosed findings, MRI participants without disclosed findings and MRI non-participants. Average treatment effects are displayed in the right column. Propensity score reweighting was applied to balance the distribution of relevant covariates between MRI participants and non-participants. Estimates were derived from two-part models. $\mathrm{N}=5019$. Left column: MRI participants with disclosed findings $\mathrm{MRI}$ participants without disclosed findings MRI non-participants. 
Primary care and prevention
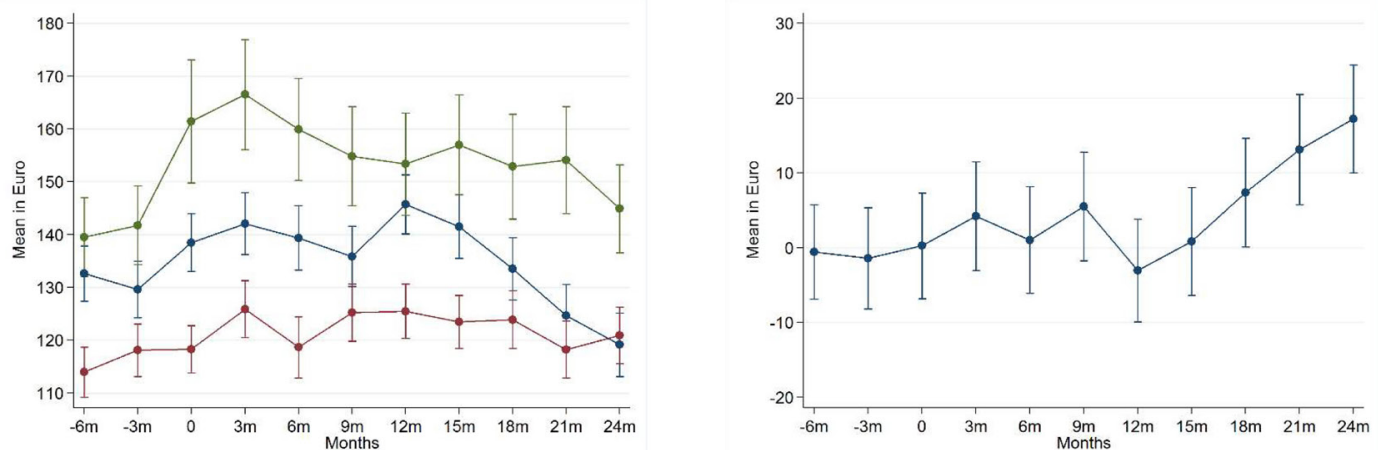

Specialist care
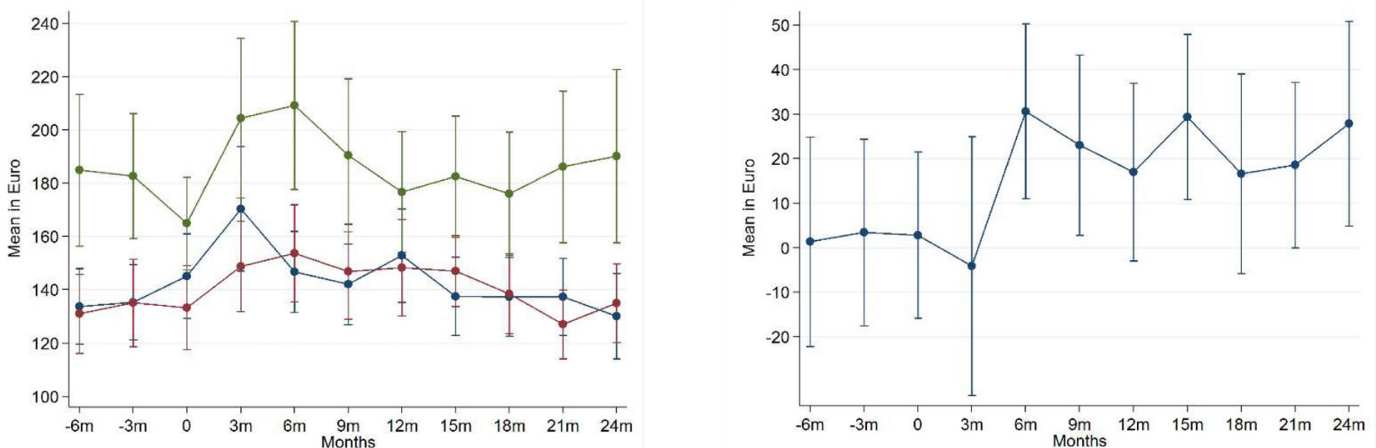

Imaging
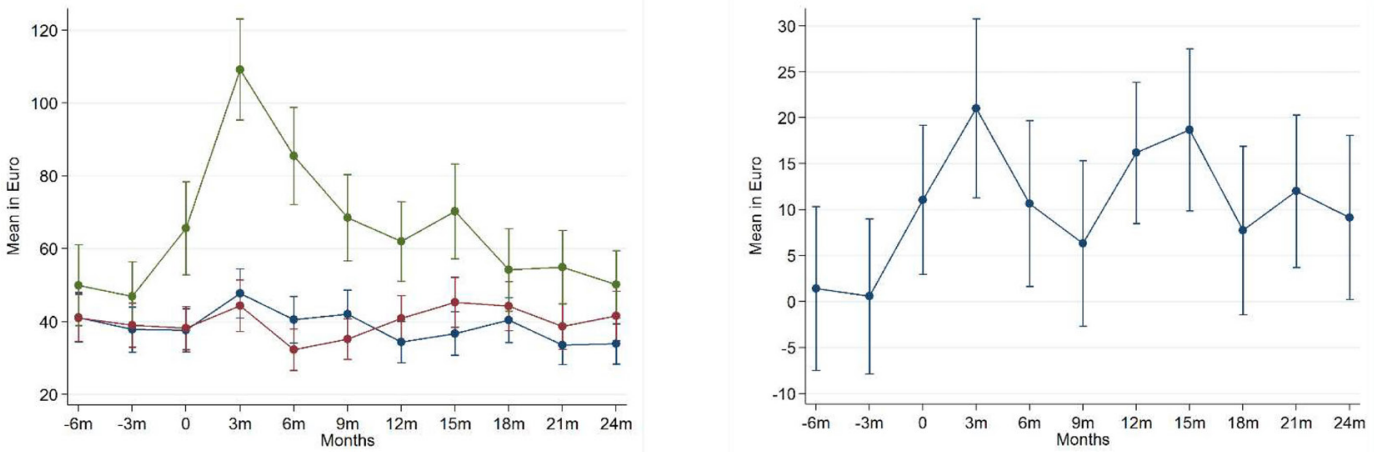

Laboratory
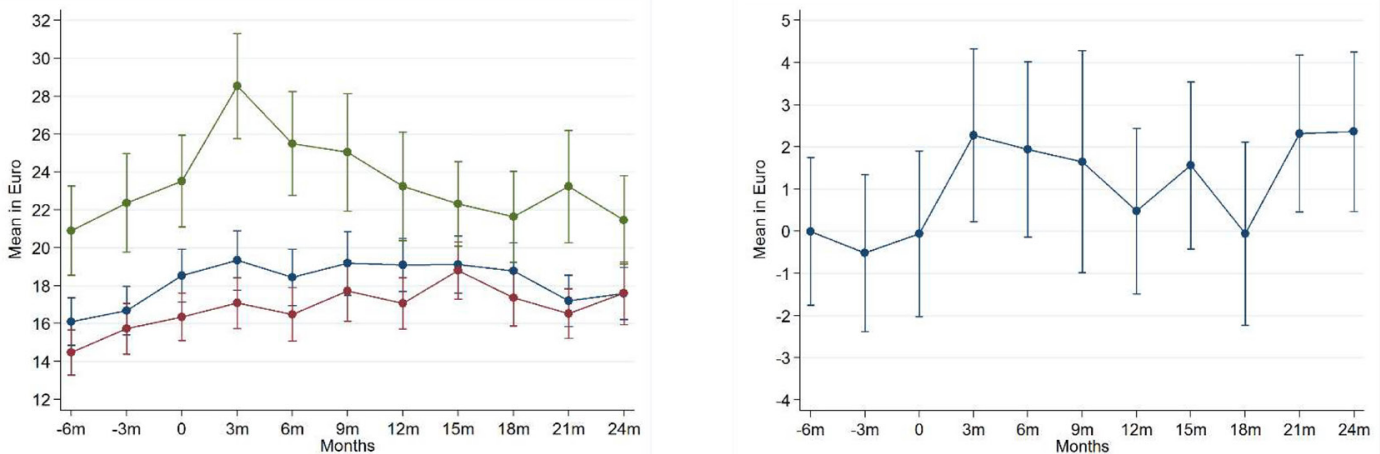

Figure 3 Course of costs by service type. "0" is the reference quarter in which the MRI examination took place. The left column displays the descriptive course for the studied outcomes comparing MRI participants with disclosed findings, MRI participants without disclosed findings and MRI non-participants. Average treatment effects are displayed in the right column. Propensity score reweighting was applied to balance the distribution of relevant covariates between MRI participants and nonparticipants. Estimates were derived from two-part models. $\mathrm{N}=5019$. Left column: $\mathrm{MRI}$ participants with disclosed findings MRI participants without disclosed findings MRI non-participants. 
Table 2 Estimated total additional costs of MRI participants compared with MRI non-participants after examination per participant in $€$

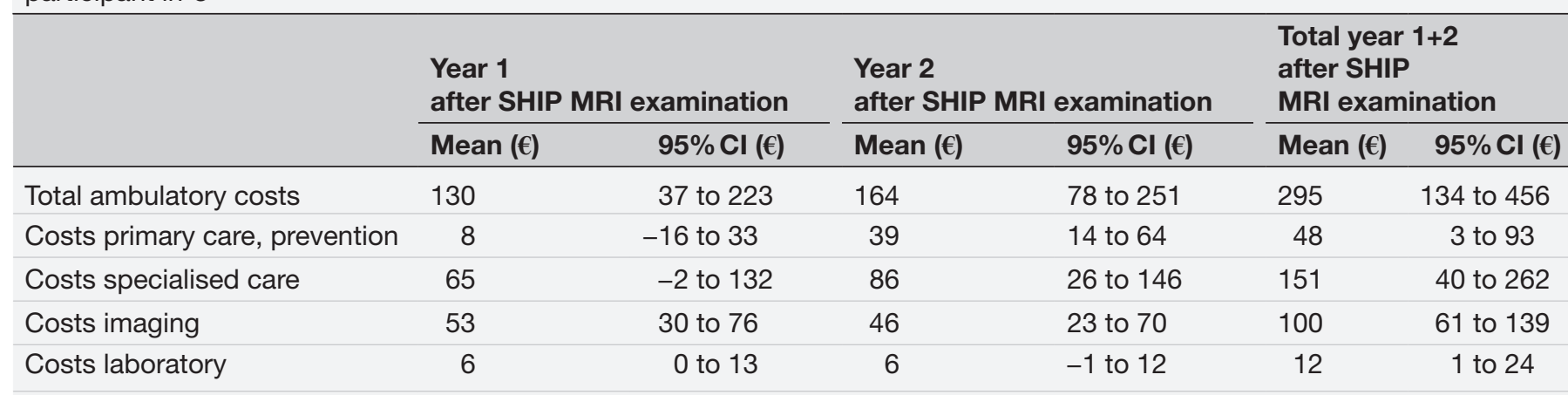

Estimates were derived from two-part models with weights to compute average treatment effects during the years 1,2 and 1+2, respectively. Year 1 comprises quarters 1-4 after the Study of Health in Pomerania (SHIP) examination. Year 2 comprises quarters 5-8 after the SHIP examinations. Year $1+2$ quarters $1-8$.

\section{MRI participation and outpatient costs}

Propensity score reweighting revealed increased excess costs among MRI participants compared with nonparticipants that persisted over time (figures 2 and 3).

Total weighted costs for outpatient care during the 2-year observation period amounted to an average of $€ 2547$ (95\% CI 2424 to 2671) for MRI non-participants and to €2839 (95\% CI 2741 to 2936) for MRI participants, yielding an average treatment effect of $€ 295$ (95\% CI 134 to 456 ), which corresponds to an increase of $+11.6 \%(5.2 \%$; $17.9 \%)$ per participant (table 2). Additional costs were higher in the second postexamination year compared with the first year. The largest contribution to excess costs resulted from specialist care, followed by imaging. The smallest effects were related to laboratory costs.

\section{DISCUSSION}

\section{Main findings}

A single wb-MRI examination sufficed to increase longterm overall outpatient costs over 2 years in a general population sample. Effects were larger for certain services such as specialist care and clinical imaging. From a research perspective, our results illustrate how disclosed IFs turned an observational study into an intervention. This limits the generalisability of research findings on outpatient costs and health service utilisation to the underlying general population. From a clinical perspective, overtesting and overdiagnosis are likely. ${ }^{3}{ }^{13}$ These results underscore in line with previous findings, ${ }^{14} 15$ that restrictive communication policies seem recommendable to protect research participants and the public from questionable clinical actions and costs while safeguarding observational research aims.

\section{Relevance from a research perspective}

We conducted a prospective cohort study without any intention to intervene. However, it was also imperative to respect our participants' health and autonomy in making healthrelated decisions. ${ }^{24}{ }^{25}$ This was deemed of particular importance for wb-MRI findings because of their potential to detect asymptomatic disease at an early, potentially treatable stage. ${ }^{1}$ The disclosure policy of wb-MRI findings in SHIP was carefully designed, but no population-based points of reference were available at that point in time. ${ }^{48}$ As a result, about $10 \%$ of all MRI findings of perceived clinical relevance in SHIP were disclosed with a recommendation for further clinical workup to almost one-third of all participants. ${ }^{8}$ As illustrated by our results, this effectively turned our cohort study into a large-scale, non-randomised intervention, converting many study participants into patients with altered outpatient care over a prolonged period. In this way, our results correspond to those of a phase I trial. ${ }^{26}$ This reduces the validity of inferences about the natural course of health services utilisation in the general population. Rather, we observed the course of outpatient care under the precondition of a populationbased health screening.

An increase in outpatient costs occurred not only in participants with disclosed MRI findings, but also in MRI nonparticipants and MRI participants without disclosed findings. This increase was comparably smaller in size and likely reflects effects of disclosures from study findings other than MRI such as laboratory results, the potential impact of which has been documented. ${ }^{14}$ Thus, carefully designed disclosure policies for research findings are needed for all study examinations, not just MRI. However, the authors do not recommend completely withholding all research findings. No disclosure at all may for example prove unethical in the rare cases that research examinations uncover severe and actionable clinical conditions.

\section{Relevance from a clinical perspective}

Participants and patients may conceivably be interested in obtaining personal health information out of a desire for reassurance about their health status or out of simple curiosity. ${ }^{167}$ However, practitioners,${ }^{28-30}$ participants and patients alike tend to overestimate the clinical relevance of findings, which is critical in the context of IFs. On one hand, similarly to other studies, we observed a large number of abnormalities on wb-MRI, the majority of them being related to tumours of an unknown nature. ${ }^{2}$ On the 
other hand, another study analysing biopsy results within the SHIP cohort found that despite the large numbers of tumour-related findings, few additional malignancies were detected. ${ }^{14}$ This is likely related to the low pretest probability of finding severe, previously undiagnosed clinical conditions in a general population sample.

Other analyses of SHIP data found that participants experienced increased psychosocial distress after the disclosure of IFs, ${ }^{16}$ yet no effects on quality of life were found $2-3$ years after the wb-MRI examination. ${ }^{15}$ This does not rule out the possibility of benefits in individual cases. Participants may also have profited from detected category III findings requiring immediate referral such as acute brain infarction or bone fracture. ${ }^{8}$ However, less than $1 \%$ of all findings of potential clinical relevance belonged to this category. Yet, it is not known if improved outcomes resulted from their communication. Furthermore, category II findings amounted to approximately only $10 \%$ of all findings. ${ }^{8}$ In SHIP, most category I findings resulted from a highly detailed structured reading protocol that also included anatomical variants. While it is unlikely that these would have been of much interest in a screening, category I findings also comprised clinical findings without any best practice recommendation to communicate them such as disc herniation. Nevertheless, in a health screening setting it seems likely that such findings would have been communicated to patients, thus leading to higher subsequent consultations and costs with an even elevated risk of overtesting, overdiagnosis and overtreatment.

Other studies support our critical view on the potential benefits of disclosed MRI findings. Evidence from randomised controlled trials support screening for only a few conditions, and none involve MRI as a screening tool. ${ }^{31}$ Moreover, the detection of a malignancy does not guarantee any clinical benefit, and may even lead to harm by overtreatment. ${ }^{3}{ }^{13}$ This is relevant for health policymakers estimating the costs and potential benefits of wb-MRI screening. The costs for the work up of IFs generated by our cohort study were covered by statutory health insurance. It is an issue of debate whether or not such findings should be subject to financing by a public health system. For example, the UK Royal College for General Practitioners argues that work up of IFs from private screening should not be the responsibility of primary care physicians. $^{32}$

\section{Strengths and limitations}

The longitudinal design, the large sample size and the availability of a control group for MRI participants are considerable strengths. The potential impact of selection bias is reduced by the low proportion of missing claims data, and the wide coverage of our participants by claims data. Yet, the small subgroup of privately insured participants $(7 \%$ in this sample) is not represented. This subgroup may be even more prone to cascades of subsequent healthcare and elevated costs due to a more favourable reimbursement of diagnostic and therapeutic measures.

Lack of data due to participants not having visited a doctor cannot be distinguished from missing data from corrupt linkage. Due to the low percentage of such cases, the expected impact on our results is low. The cost of performing the wb-MRI itself is not included in our analyses.

Participants in general-population cohort studies have been found to show fewer unhealthy behaviours, enhancing the generalisability of our findings to screening initiatives which cater to persons seeking ${ }^{18}$ such services but also to other general populations health studies.

The observational nature of SHIP limits causal inferences. Yet, given the observed temporal patterns, the markedly different course of outpatient costs among MRI participants with disclosed findings compared with those without leaves little plausible options for alternative explanations underlying the observed increases in costs. ${ }^{16}$ While being closely related to health service costs, the frequency of consultations cannot be validly inferred from German claims data. Therefore, despite being of interest, this aspect was not addressed.

We targeted the impact of communicated MRI findings and computed related treatment effects. However, other clinical findings were disclosed as well such as laboratory results. These were not examined independently in our study. Increases in healthcare costs in participants without disclosed MRI IFs (figures 2 and 3) indicate that elevated costs were potentially related to the disclosure of such findings. However, effect sizes are much smaller compared with those in participants with disclosed MRI IFs.

Because available claims data do not cover medication costs or inpatient care as well as emergency department attendances, ${ }^{33}$ we likely underestimate the increase in total healthcare-related expenditures. However, it is unlikely that the inclusion of inpatient costs would have substantially altered our conclusions because diagnostics for IFs rarely justify hospital admission. Given our research setting, we also likely underestimate health service utilisation and costs resulting from clinically indicated wb-MRI, given the patient's right to disclosure of all IFs in a clinical setting. ${ }^{4}$

\section{Conclusion}

wb-MRI examination in a general population sample has sustained effects on health service utilisation, leading to elevated costs that may well persist beyond the duration of the 2-year observation period after the wb-MRI examination. The disclosure of IFs in this prospective cohort study biased the longitudinal study of health related outcomes, generated additional costs to the public, and likely induced overdiagnosis and overtesting.

\section{Author affiliations}

${ }^{1}$ Institute for Community Medicine - Department SHIP/KEF, University Medicine Greifswald, Greifswald, MV, Germany

${ }^{2}$ Institute for Community Medicine - Department of Family Medicine, University Medicine Greifswald, Greifswald, MV, Germany

${ }^{3}$ Institute of Health Services Research in Dentistry, University of Muenster, Muenster, Niedersachsen, Germany

${ }^{4}$ Unfallkrankenhaus Berlin, Berlin, Germany

${ }^{5}$ Department of Diagnostic Radiology and Neuroradiology, University Medicine Greifswald, Greifswald, MV, Germany 
${ }^{6}$ DZD - German Center for Diabetes Research - Partner Site Greifswald, Greifswald, MV, Germany

${ }^{7}$ DZHK - German Centre for Cardiovascular Research - Partner Site Greifswald, Greifswald, MV, Germany

\section{Twitter Jean-François Chenot @F-8241-2011}

Contributors COS conceived the study, supervised the data linkage, conducted statistical analyses, and is responsible for the overall content as guarantor. $\mathrm{HV}$ is $\mathrm{PI}$ of the SHIP study, KH was in charge of the SHIP-MRI. J-FC, ES provided expertise for claims data selection and analysis. SB was involved in the study analysis and provided statistical and epidemiological expertise, and approved the manuscript. COS, J-FC and ES wrote the first draft which was revised and approved by all authors.

Funding This work was specifically funded by the German Research Foundation [SCHM 2744/1-1/1-2 to COS and CH 921/1-2 to JFC]; the SHIP study was funded by the Federal Ministry of Education and Research [03ZIK012], the Ministry of Cultural Affairs; the Social Ministry of the Federal State of MecklenburgVorpommern; Siemens Healthcare, Erlangen, Germany and Bayer Healthcare.

Disclaimer The funders did not play any role in the study design, data collection and analysis, decision to publish, or preparation of the manuscript.

Competing interests None declared.

Patient consent for publication Not applicable.

Ethics approval The Ethics Committee of the University Medicine of Greifswald approved the study protocol (BB 39/08, BB 106/10). All participants provided written informed consent before being recruited into SHIP.

Provenance and peer review Not commissioned; externally peer reviewed.

Data availability statement Data are available upon reasonable request. Data of the SHIP studies are available and can be applied for under https://www.fvcm.med. uni-greifswald.de/dd_service/data_use_intro.php. The claims data are not publicly available due to privacy restrictions and legal reasons.

Open access This is an open access article distributed in accordance with the Creative Commons Attribution Non Commercial (CC BY-NC 4.0) license, which permits others to distribute, remix, adapt, build upon this work non-commercially, and license their derivative works on different terms, provided the original work is properly cited, appropriate credit is given, any changes made indicated, and the use is non-commercial. See: http://creativecommons.org/licenses/by-nc/4.0/.

\section{ORCID iDs}

Carsten Oliver Schmidt http://orcid.org/0000-0001-5266-9396 Jean-François Chenot http://orcid.org/0000-0001-8877-2950

\section{REFERENCES}

1 Orme NM, Fletcher JG, Siddiki HA. Incidental findings in imaging research: evaluating incidence, benefit, and burden. Arch Intern Med 2010;170:1525-32

2 Kwee RM, Kwee TC. Whole-body MRI for preventive health screening: a systematic review of the literature. J Magn Reson Imaging 2019;50:1489-503.

3 Moynihan R, Doust J, Henry D. Preventing overdiagnosis: how to stop harming the healthy. BMJ 2012;344:e3502.

4 Booth TC, Waldman AD, Wardlaw JM, et al. Management of incidental findings during imaging research in "healthy" volunteers: current UK practice. Br J Radiol 2012;85:11-21.

5 Berlin L. How do you solve a problem like incidentalomas? Appl Radiol 2013:42:10-12.

6 Bamberg F, Kauczor H-U, Weckbach S, et al. Whole-body MR imaging in the German national cohort: rationale, design, and technical background. Radiology 2015;277:206-20.

7 Völzke H, Alte D, Schmidt CO, et al. Cohort profile: the study of health in Pomerania. Int J Epidemiol 2011;40:294-307.

8 Hegenscheid K, Seipel R, Schmidt CO, et al. Potentially relevant incidental findings on research whole-body MRI in the general adult population: frequencies and management. Eur Radiol 2013;23:816-26.
9 Tarnoki DL, Tarnoki AD, Richter A, et al. Clinical value of whole-body magnetic resonance imaging in health screening of general adult population. Radiol Oncol 2015;49:10-16.

10 O'Sullivan JW, Muntinga T, Grigg S, et al. Prevalence and outcomes of incidental imaging findings: umbrella review. BMJ 2018;361:k2387.

11 Gibson LM, Paul L, Chappell FM, et al. Potentially serious incidental findings on brain and body magnetic resonance imaging of apparently asymptomatic adults: systematic review and metaanalysis. BMJ 2018;363:k4577.

12 Welch HG, Black WC. Overdiagnosis in cancer. J Natl Cancer Inst 2010;102:605-13.

13 Brodersen J, Kramer BS, Macdonald H, et al. Focusing on overdiagnosis as a driver of too much medicine. BMJ 2018;362:k3494.

14 Richter A, Sierocinski E, Singer S, et al. The effects of incidental findings from whole-body MRI on the frequency of biopsies and detected malignancies or benign conditions in a general population cohort study. Eur J Epidemiol 2020;35:925-35.

15 Schmidt CO, Sierocinski E, Hegenscheid K, et al. Impact of whole-body MRI in a general population study. Eur J Epidemiol 2016;31:31-9.

16 Schmidt CO, Hegenscheid K, Erdmann P, et al. Psychosocial consequences and severity of disclosed incidental findings from whole-body MRI in a general population study. Eur Radiol 2013;23:1343-51.

17 Evans $\mathrm{A}$, Tolonen $\mathrm{H}$, Hense $\mathrm{HW}$, et al. Trends in coronary risk factors in the who MONICA project. Int J Epidemiol 2001;30:S35-40.

18 Schmidt CO, Alte D, Völzke H, et al. Partial misspecification of survey design features sufficed to severely bias estimates of health-related outcomes. J Clin Epidemiol 2011;64:416-23.

19 Hegenscheid K, Kühn JP, Völzke $\mathrm{H}$, et al. Whole-body magnetic resonance imaging of healthy volunteers: pilot study results from the population-based SHIP study. Rofo 2009;181:748-59.

20 Belotti F, Deb P, Manning WG, et al. Twopm: two-part models. Stata J 2015;15:3-20.

21 Guo S, FM W. Propensity Score Analysis: Statistical Methods and Applications. Los Angeles: SAGE, 2009.

22 Nichols A. Erratum and discussion of propensity score reweighting. Stata J 2008;8:532-9.

23 Busija L, Pausenberger E, Haines TP. Adult measures of general health and health-related quality of life: medical outcomes study short form 36-Item (SF-36) and short form 12-Item (SF-12) health surveys, Nottingham health profile (NHP), sickness impact profile (SIP), medical outcomes study short form 6D (SF-6D), health Utilities index mark 3 (HUI3), quality of well-being scale (QWB), and assessment of quality of life (AQoL). Arthritis Care Res 2011;63:S383-412.

24 DeFrank JT, Barclay C, Sheridan S, et al. The psychological harms of screening: the evidence we have versus the evidence we need. J Gen Intern Med 2015;30:242-8.

25 Viberg J, Hansson MG, Langenskiöld S, et al. Incidental findings: the time is not yet ripe for a policy for biobanks. Eur J Hum Genet 2014;22:437-41.

26 Pinato DJ, Stavraka C, Tanner M, et al. Clinical, ethical and financial implications of incidental imaging findings: experience from a phase I trial in healthy elderly volunteers. PLoS One 2012;7:e49814.

27 Townsend A, Cox SM. Accessing health services through the back door: a qualitative interview study investigating reasons why people participate in health research in Canada. BMC Med Ethics 2013;14:40.

28 Sajid IM, Parkunan A, Frost K. Unintended consequences: quantifying the benefits, iatrogenic harms and downstream cascade costs of musculoskeletal MRI in UK primary care. BMJ Open Qual 2021;10:e001287.

29 Morgan DJ, Pineles L, Owczarzak J, et al. Accuracy of practitioner estimates of probability of diagnosis before and after testing. JAMA Intern Med 2021;181:747-55.

30 Whiting PF, Davenport C, Jameson C, et al. How well do health professionals interpret diagnostic information? A systematic review. BMJ Open 2015;5:e008155.

31 Saquib N, Saquib J, loannidis JPA. Does screening for disease save lives in asymptomatic adults? Systematic review of meta-analyses and randomized trials. Int J Epidemiol 2015;44:264-77.

32 Mahase E. Private screening: GPs shouldn't have to deal with results, says RCGP. BMJ 2019;366:15707.

33 Swart E, Ihle P, Gothe H. Routinedaten im Gesundheitswesen: Handbuch Sekundärdatenanalyse: Grundlagen, Methoden und Perspektiven. Bern: Hogrefe, 2014. 